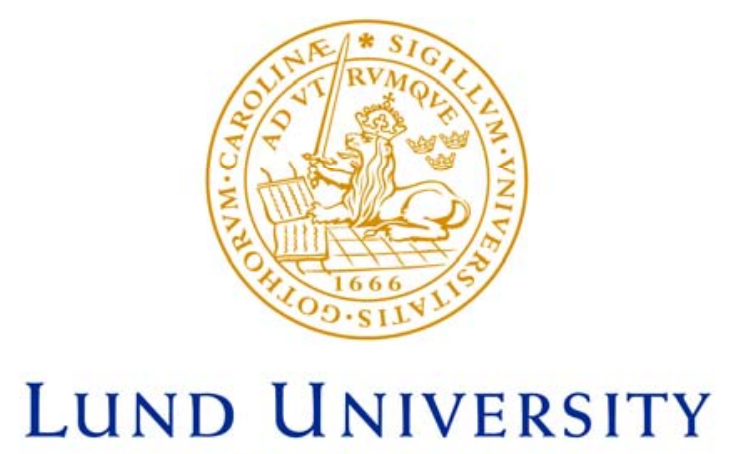

Faculty of Medicine

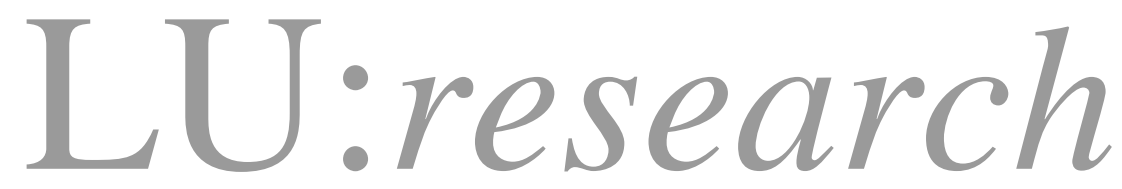

Institutional Repository of Lund University

This is an author produced version of a paper published in The Journal of allergy and clinical immunology. This paper has been peer-reviewed but does not include the final publisher proof-corrections or journal pagination.

Citation for the published paper:

Persson, Helena and Sadegh, Mardjaneh Karbalaei and Greiff, Lennart and Ohlin, Mats.

"Delineating the specificity of an IgE-encoding transcriptome"

J Allergy Clin Immunol, 2007, Sep 6 [Epub ahead of print]

http://dx.doi.org/10.1016/j.jaci.2007.06.041

Access to the published version may

require journal subscription.

Published with permission from: Elsevier 


\section{Delineating the specificity of an IgE-encoding transcriptome}

Helena Persson, MSc, ${ }^{\text {a }}$ Mardjaneh Karbalaei Sadegh, MSc, ${ }^{a}$ Lennart Greiff, MD, ${ }^{\text {a }}$ and Mats Ohlin, $\mathrm{PhD}^{\mathrm{a} *}$

${ }^{\mathrm{a} D e p a r t m e n t ~ o f ~ I m m u n o t e c h n o l o g y, ~ L u n d ~ U n i v e r s i t y, ~ B M C ~ D 13, ~ S E-221 ~} 84$ Lund, Sweden

${ }^{\mathrm{b}}$ Department of Otorhinolaryngology, Lund University Hospital, SE-221 85 Lund, Sweden

*To whom correspondence should be addressed:

E-mail address: mats.ohlin@immun.lth.se

Tel: +46-46-222 4322

Fax: +46-46-222 4200

Funding: The Swedish Asthma and Allergy Association's Research Foundation, Alfred Österlunds Foundation and the Swedish Research Council.

Total word count: 3594 


\section{Abstract}

Background: Although much is known about the reactivity of polyclonal populations of antibodies targeting the wide array of allergens produced by timothy (Phleum pratense) and other grass species, little is known about the finer details at the level of individual antibody specificities.

Objective: We sought to investigate the IgE repertoire as it occurs in a grass pollen allergic patient.

Methods: For this purpose, a human IgE library, constructed from peripheral blood B-cells of a timothy allergic individual, was used. The library was screened, using phage display, against a panel of six different timothy allergens (Phl p 1, Phl p 2, Phl p 4, Phl p 5, Phl p 6, and Phl p 11).

Results: Highly diverse antibody fragments with respect to gene usage were identified. The binders were highly specific for their respective target antigen, except for clones selected on Phl p 6 which also recognized Phl p 5, most likely reflecting the high sequence homology between these allergens. Interestingly, by using this approach we were able to determine the specificity of over $25 \%$ of all IgE-producing transcripts in this allergic individual.

Conclusion: The human IgE repertoire is produced by a limited number of highly related Bcell clones and as such restricted in its recognition of a limited number of antigens.

Clinical Implication: Human allergen-specific antibodies can, by defining the specificity of IgE responses, aid in the development of allergy vaccines or even by themselves be used in passive immunotherapy. 
Accepted for publication in the Journal of Allergy and Clinical Immunology (2007)

DOI:10.1016/j.jaci.2007.06.041

Capsule summary: Findings from this study demonstrate that just a few allergens account

for a high percentage of the complex IgE repertoire in a grass-pollen allergic patient, a factor of importance for the development of allergy therapies.

Key words: allergy, antibody library, antibody repertoire, diversity, grass pollen allergen, IgE, phage display, specificity 
Accepted for publication in the Journal of Allergy and Clinical Immunology (2007) DOI:10.1016/j.jaci.2007.06.041

Abbreviations

BSA: bovine serum albumin

CDR: complementarity determining region(s)

HRP: horse-radish peroxidase

PBS: phosphate buffer saline

scFv: single chain antibody fragment(s)

$\mathrm{V}_{\mathrm{H}}$ : variable region of the antibody heavy chain

$\mathrm{V}_{\mathrm{L}}$ : variable region of the antibody light chain 


\section{Accepted for publication in the Journal of Allergy and Clinical Immunology (2007)}

DOI:10.1016/j.jaci.2007.06.041

\section{INTRODUCTION}

Grass pollen allergy has been estimated to affect up to $20 \%$ of the population in the industrialized countries, ${ }^{1,2}$ consequently affecting the quality of life of millions of people. The disease mechanism is associated with increased serum-levels of specific IgE that are mainly directed towards a limited number of proteins that are released from the pollen grain upon hydration. Binding of these allergens to basophil- and mast cell-bound IgE leads to Fcreceptor cross-linking, degranulation and the release of inflammatory mediators which in turn causes the symptoms associated with grass pollen allergy, ranging from rhinitis and conjunctivitis to asthma. As the antibody-allergen interaction is a key event in the allergic reaction, a deeper understanding of it is a prerequisite for understanding the mechanisms behind allergy and such knowledge will also aid in the development of new allergy treatments.

Although much is known about the make-up of genes encoding antibodies in general and also to some extent of genes encoding IgE, much less is known about sequences of antibodies targeting specific allergens. In a previous investigation, ${ }^{3}$ we set out to study the IgE repertoire as it occurs in grass-pollen allergic patients. Sequencing the heavy chain of random transcripts of this repertoire, we found these antibody populations to highly resemble immunoglobulin populations of other isotypes with respect to genetic diversity and mutational patterns, a finding supported ${ }^{4-6}$ as well as contradicted ${ }^{7-10}$ by other studies. However, in

contrast to IgG populations, ${ }^{11}$ a high number of clonally related sequences were identified among the analyzed clones, suggesting that despite its rather diverse genetic make-up the peripheral IgE population is populated by a limited set of related clones, or so called clonotypes. This latter finding confirms the results of previous studies. ${ }^{4,12,13}$ Taken together, this lead us to hypothesize that the relatively small IgE population of allergic individuals is limited in its recognition of just a few allergens at a given time. In order to define the 


\section{Accepted for publication in the Journal of Allergy and Clinical Immunology (2007)}

DOI:10.1016/j.jaci.2007.06.041

specificities of the identified clonotypes, a combinatorial phage display library was

constructed from the IgE repertoire of the patients mentioned above and selections were initially performed on the major grass pollen allergen $\mathrm{Phl} \mathrm{p} \mathrm{5.}{ }^{3}$ Highly specific antibody fragments, binding to several different epitopes on Phl p 5, were identified. Interestingly, one of the selected binders was highly similar to a set of sequences found among the randomly picked IgE-encoding transcripts. Numerous studies have explored the reactivity of polyclonal IgE populations to many different allergens, including those of grass pollen, but when it comes to the reactivity pattern of individual IgE-molecules data is limited. ${ }^{14-18}$ In contrast to our investigation, ${ }^{3}$ however, none of the previous studies have described the antibodies within the larger context of the whole IgE repertoire. Our combined approach of both studying the overall IgE repertoire and the IgE repertoire targeting specific allergens therefore put us in a unique position, allowing us to investigate the specificity profile of this population at a much higher resolution and further providing us with new insights on how allergen-specific responses arise.

In this study, we have continued on the path of determining the specificity profile of the IgE-encoding repertoire of a grass-pollen allergic patient. We show that 6 timothy pollen allergens (Phl p 1, Phl p 2, Phl p 4, Phl p 5, Phl p 6, and Phl p 11) account for over 25\% of the IgE producing transcripts of this donor, further supporting the notion of IgE repertoires having limited recognition ranges. 


\section{METHODS}

\section{IgE-scFv library}

The local ethical committee gave approval for the procedures in this study. The construction of the combinatorial single chain antibody fragment (scFv) library used has previously been described. ${ }^{3}$ Briefly, peripheral blood lymphocytes had been isolated from a grass pollen allergic donor, A3, during season of antigen exposure (June) as part of a study investigating immunological principles in allergy. ${ }^{19}$ Except for showing reactivity in clinical skin-prick testing to timothy extract, the patient also had clear symptoms of rhinitis and had high levels of timothy extract specific IgE (55 kUA/l) at the time of sampling. Additionally, skin prick testing on A3 revealed sensitivity to cat and birch. ${ }^{19}$ The heavy $\left(\mathrm{V}_{\mathrm{H}}\right)$ and light chain $\left(\mathrm{V}_{\mathrm{L}}\right.$ ) encoding genes were obtained by a semi-nested PCR approach, the $\mathrm{V}_{\mathrm{H}}$ genes having an origin in the IgE repertoire, while the $\mathrm{V}_{\mathrm{L}}$ genes were derived from $\mathrm{B}$ cells producing different antibody isotypes. The $\mathrm{V}_{\mathrm{H}}$ and $\mathrm{V}_{\mathrm{L}}$ encoding genes were assembled using overlap-extension PCR and subsequently incorporated into a modified form of the pFab5c.His phagemide. ${ }^{20}$ The ligation product was transformed into electrocompetent Top10F’ (Invitrogen, Carlsbad, CA, USA), giving a library size of approximately $3 \times 10^{5}$ clones. The library was infected by VCSM13 helper phage (Stratagene, La Jolla, CA, USA) and phage harvested by standard polyethylene glycol/ $\mathrm{NaCl}$ precipitation.

\section{Phage display selections of antibody fragments specific for timothy allergens}

To identify timothy-specific IgE, phage selections were performed on 7 different allergens from timothy grass (Phleum pratense); Phl p 1, Phl p 2, Phl p 4, Phl p 5 (isoform a), Phl p 6, Phl p 7 and Phl p 11. All antigens were purchased from Biomay AG (Vienna, Austria) except for Phl p 4 and Phl p 11, which were kindly provided by Dr. Jonas Lidholm (Phadia AB, Uppsala, Sweden). Recombinant versions of the allergens were used, except in 


\section{Accepted for publication in the Journal of Allergy and Clinical Immunology (2007)}

DOI:10.1016/j.jaci.2007.06.041

the case of Phl p 4 where the natural form was utilized. Selections were performed in microtiter plates (Costar, Cambridge, MA, USA) by first coating the plates with allergens diluted to $5 \mu \mathrm{g} / \mathrm{ml}$ in phosphate buffer saline (PBS). Unbound antigen was washed away and blocking solution (1\% (w/v) bovine serum albumin (BSA), 0.05\% Tween $20(\mathrm{v} / \mathrm{v})$ in PBS) added before the phage library, described above, was applied. After washing, unbound phages were eluted by the addition of trypsin, which cleaves at a site located between the displayed scFv and protein III. After two and/or three rounds of selections, phage stocks were produced from individual randomly picked clones and analyzed for binding specificity. The clones were named with a prefix indicating the antigen selected on ( $\mathrm{p} 1$ for Phl p 1, p2 for Phl p 2, etc.) followed by a clone number.

\section{ELISA analysis}

The phage-displayed scFv were analyzed for binding specificity by phage ELISA. The allergens, BSA and extracts of Phleum pratense and Lolium perenne (both extracts were kindly provided by P. A. Würtzen, ALK-Abelló, Hørsholm, Denmark) were coated in microtiter plates at a concentration of $5 \mu \mathrm{g} / \mathrm{ml}$. Skim milk was used as blocking agent (1\% (w/v) milk, 0.05\% Tween 20 (v/v) in PBS) and bound phage were detected by a horse-radish peroxidase (HRP) conjugated anti-M13 monoclonal antibody (GE Healthcare, Uppsala, Sweden) using o-phenylenediamine as chromogen. Absorbance was measured at $490 \mathrm{~nm}$. The clones were considered positive if the absorbance for their respective target antigens was at least 6 times higher than that obtained for control antigens, like BSA.

The reactivity of donor A3's IgE to the different allergens used during the selections as well as to recombinant Phl p 12 (a kind gift from Dr. Jonas Lidholm, Phadia AB) was verified by ELISA analysis performed on serum aliquots from this donor, collected during season of grass pollen exposure (June). Bound IgE was detected with the HRP-labeled goat-anti-human 
IgE (KPL Inc., Gaithersburg, Maryland, USA). Coating, blocking and detection were performed as described above.

\section{Genetic analysis}

The allergen-specific clones were subjected to DNA sequencing (MWG Biotech, Ebersberg, Germany). The genetic origin of their $\mathrm{V}_{\mathrm{H}}$, excluding the bases that were part of the primer definition when constructing the library, ${ }^{3}$ were analyzed by the bioinformatics tools IMGT/V-Quest $^{21}$ and IMGT/Junction ${ }^{22}$ (http://imgt.cines.fr/). The $\mathrm{V}_{\mathrm{H}}$ sequences of the selected clones were also compared to previously described Phl p 5-specific scFv and to randomly sequenced IgE VH sequences, all with an origin in the A3 donor. ${ }^{3}$ Clones were considered clonally related if having an origin in the same IGHV gene and having a highly homologous CDRH3, having identical length (between C104 and W118) and sharing at least $80 \%$ similarity on nucleotide level. Throughout this article, the IMGT nomenclature is used for complementarity determining region (CDR) definitions and residue numbering. ${ }^{23}$ 


\section{RESULTS}

\section{IgE-reactivity profile to different timothy allergens}

The combinatorial IgE library used in this study had been derived from a donor, A3, which previously had been tested positive in skin prick testing to timothy extract. ${ }^{19}$ In addition, serum from this donor was tested in an ELISA for the presence of IgE antibodies specific to the individual timothy allergens (Phl p 1, Phl p 2, Phl p 4, Phl p 5, Phl p 6, Phl p 7 , Phl p 11 and Phl p 12) used in this study (Figure 1). Reactive antibodies were found in all cases except for Phl p 7 and Phl p 12. The lack of reactivity to these two allergens is not surprising since only around 10-20\% of grass-pollen allergic individuals have an IgE response against them. . $2^{2}$

\section{Selection of scFv specific for different timothy allergens}

The IgE library was subjected to phage selections on 7 different timothy allergens (Phl p 1, Phl p 2, Phl p 4, Phl p 5, Phl p 6, Phl p 7 and Phl p 11). In most cases, high output/input ratios could initially be observed, underscoring the high frequency of specific binders in the library. Following two or three rounds of selections, randomly picked clones were analyzed for specificity using phage-ELISA (Figure 2). Consistent with the IgE serum data we were able to isolate binders to all of the antigens except for $\mathrm{Phl} \mathrm{p} \mathrm{7,} \mathrm{demonstrating} \mathrm{that} \mathrm{the} \mathrm{selected}$ binders most likely had a counterpart in vivo. The isolated binders were highly specific to their respective target allergen, except those selected on Phl p 6, which also bound Phl p 5, an allergen with extensive sequence homology to Phl p 6. ${ }^{24,25}$

Most of the isolated scFv also bound the natural form of the antigen as shown by the observed reactivity, although of various strengths, with pollen extracts of timothy and/or rye grass (Figure 2). In most cases these species produce similar allergens, which explain why both extracts can be recognized. An exception is Phl p 6, which have been identified in 
timothy but not in rye grass. ${ }^{26}$ The obtained signal to rye grass of the Phl p 6-selected clones is therefore likely due to the above mentioned cross-reactivity resulting in binding to the group 5 allergens of this extract. The low signal that was observed for the Phl p 11-specific scFv does not necessarily mean that these binders do not recognize the natural antigen, but may reflect a low concentration of this allergen in the extracts. Furthermore, a potential glycosylation site found in this allergen might possible explain the observed differences. ${ }^{27}$

\section{Clonal relationship of the heavy chains of the IgE-encoding repertoire}

Our previous analysis of the IgE transcriptome of two donors ${ }^{3}$ indicated that few clonotypes populate the IgE-encoding repertoire, i.e. multiple related sequences were identified when sequencing these transcriptomes. For example, among the 63 randomly sequenced $\mathrm{V}_{\mathrm{H}}$ transcripts of donor A3, only 33 different clonotypes were identified (Table I, clonotype 1-33), suggesting that many of the clones had an origin in the same precursor clone.

The allergen-specific clones isolated in this study could similarly be organized into clonotypes based on heavy chain sequence homology (sharing the same IGHV gene and having highly similar CDRH3 sequences). Altogether, 12 different clonotypes, with an origin in all major IGHV subgroups (1, 3, 4 and 5), were identified (Table I). Except in the case of Phl p 2, at least two clonotypes for each allergen target were found, utilizing diverse sets of genes, demonstrating that the IgE response against these allergens are not clonally restricted.

When comparing sequences of the allergen-specific scFv with the randomly picked IgEencoding transcripts, obtained from the donor (A3) who contributed the sequences of the library, it was shown that 5 of the 12 clonotypes were found in both sets of sequences (Table I, clonotype 5, 18 and 24-26). Two Phl p 5-specific clones have additionally, in two separate studies $^{3}$ (J. Persson et al., manuscript in preparation), been identified among the random transcripts (Table I, clonotype 16 and 29) using the same library, bringing the total number of 
clonotypes with a defined allergen specificity to 14 (Table II). 7 of the clonotypes, including the three Phl p 6-selected clonotypes, could not be found among the previously described clonotypes (Table I, clonotype 34-40), suggesting that they were minor components of the IgE-encoding transcriptome.

Overall, it was shown that the repertoire targeting just 5 allergens (Phl p 1, Phl p 2, Phl p 4, Phl p 5 and Phl p 11) alone covered 21-27\%, 7 of 33 clonotypes or 17 of 63 members, of the IgE $\mathrm{V}_{\mathrm{H}^{-}}$-encoding transcriptome as represented by randomly sequenced transcripts. This implies that just a few allergens elicit the IgE repertoire encoded by circulating B cells at a given point in time.

\section{The light chain of selected allergen-specific antibodies}

The combination of combinatorial library and display technologies like phage display is commonly exploited to investigate specific antibody repertoires. The combinatorial principles of library construction, i.e. the random assortment of light chains with heavy chains in proteins encoded for by the library, however, adds diversity to the population that does not have a counterpart in the repertoire as found in vivo. In addition, the library used in this study utilized light chains from antibodies of all isotypes, not just restricted to the IgE population, thereby increasing the diversity even further. Having this in mind, one would assume the selected scFv to be highly diverse in the choice of light chains. However, this was generally not the case. All but one of the allergen-specific $\mathrm{V}_{\mathrm{H}}$ clonotypes that were selected in multiple copies, were highly restricted in the choice of light chain partner, i.e. the selected binders used very similar light chain sequences (Table II). Altogether, these results strongly suggest that an allergen-specific binder derived from the $\mathrm{V}_{\mathrm{H}}$ of these clones in general could only be formed upon combination with a very restricted set of $V_{L}$. 


\section{Accepted for publication in the Journal of Allergy and Clinical Immunology (2007) DOI:10.1016/j.jaci.2007.06.041}

\section{DISCUSSION}

In a previous study, ${ }^{3}$ we defined the IgE repertoire as it occurs in a grass pollen sensitized individual. Among a limited set of randomly sequenced IgE $\mathrm{V}_{\mathrm{H}}$ transcripts highly related sequences were identified. These and similar results obtained in other studies ${ }^{4,12,13}$ indicate that the peripherally located IgE-producing B-cell population is populated by a relatively restricted set of clones. The 63 clones that were analyzed could based on sequence homology, i.e. sharing the same IGHV and having highly similar CDRH3 loops, be clustered into 33 different groups or clonotypes (Table I). Interestingly, among the Phl p 5-specific scFv that were isolated from the combinatorial phage library constructed from the IgE repertoire of the same donor, a scFv belonging to one of these 33 clonotypes was retrieved (Table I, clonotype 29). By fusing the light chain of this Phl p 5-specific antibody fragment to the $\mathrm{V}_{\mathrm{H}}$ of two other members of clonotype 29, antibodies with retained allergen specificity were created, thus suggesting that clones belonging to the same clonotype most likely have

the same specificity. ${ }^{3}$ This is consistent with the fact that the heavy chain, especially CDRH3, play a key role in antigen recognition. ${ }^{28}$ The differences observed between members of the same $\mathrm{V}_{\mathrm{H}}$ clonotype likely describe different evolutionary pathways obtained as a result of the affinity maturation process to the antigen in question.

Encouraged by these results, we have now embarked on a journey with the aim of trying to obtain a more complete picture of the specificity of the IgE repertoire. In the current study we have, using the same library, identified antibody fragments with specificity to additional five timothy allergens (Phl p1, Phl p 2, Phl p 4, Phl p 6, Phl p 11). As in the case of the Phl p 5-specific antibody fragments, many of the newly selected binders belonged to clonotypes previously identified among the IgE $\mathrm{V}_{\mathrm{H}}$ transcripts. ${ }^{3}$ Although the specificities have not been proven for the randomly picked $\mathrm{V}_{\mathrm{H}}$ transcripts, it may be assumed, based on the above reasoning, that they represent the same specificity as their phage-selected homologues. 


\section{Accepted for publication in the Journal of Allergy and Clinical Immunology (2007)}

DOI:10.1016/j.jaci.2007.06.041

Altogether, by using this procedure it was shown that the repertoire targeting this restricted set of allergens alone covered around 25\% of the IgE-encoding transcriptome. Previously, absorbent studies on polyclonal populations have similarly concluded a limitation in the number of recognized allergens by IgE repertoires. ${ }^{29,}{ }^{30}$ However, the allergen-specific antibody fragments as identified and characterized here give an important insight into the way the immune system recognizes the allergen at a level of resolution reaching beyond that achievable by assessing polyclonal antibody repertoires as found in the serum. The current study provides a picture of the immune response during a single time-point during allergen peak season and it is therefore unclear whether the identified population is a snapshot in time of an evolving response or if they represent a constant final set of clones. Niederberger et al. ${ }^{31}$ recently suggested that allergen exposure leads to the activations of an established pool of IgE memory cells rather than an activation of immature B-cells, indicating that the IgE producing population is rather fixed over time. However, the existence of an IgE memory B cell compartment has been questioned ${ }^{32}$ and further investigations regarding IgE plasma cell homeostasis is needed in order to shed further light on the subject.

The isolated binders were highly specific for their respective target antigen except those obtained from the Phl p 6 selections, which showed high cross-reactivity with Phl p 5. These allergens are highly homologous, having regions of over $90 \%$ sequence identity. ${ }^{24,}{ }^{25}$ Identical or over-lapping epitopes between the two allergens are likely to be the reason for the observed cross-reactivity. These findings demonstrate the close immunological similarity between Phl p 5 and Phl p 6 and support, at a clonal level, the notion that epitopes found on Phl p 6 have a counterpart in Phl p 5. ${ }^{1,2}$

Since combinatorial libraries randomly recombine heavy and light chains one might ask if the antibodies selected from such libraries constitute a valid representation of the in vivo immune response. Importantly, as demonstrated by the inability of identifying Phl p 7- 


\section{Accepted for publication in the Journal of Allergy and Clinical Immunology (2007)}

DOI:10.1016/j.jaci.2007.06.041

specific scFv, an allergen for which the donor had no measurable serum IgE, the

combinatorial process as such did not create novel specificities that had no counterpart in vivo. Quite contrary, the sequences of many of the allergen-specific scFv of the current study suggest that the pairing could have developed in B-cells. A predominance of certain $\mathrm{V}_{\mathrm{H}}-\mathrm{V}_{\mathrm{L}}$ pairs, particularly involving different but highly related clones, suggests that the observed combinations are associated with antigen binding in vivo. This was very apparent for sequences belonging to clonotype 26 encoding $\mathrm{Phl} \mathrm{p} 2$ specificity. This common sequence was only selected in combination with light chain sequences originating from the two highly related germline genes IGKV1-5 and IGKV1-12 (Table II). Furthermore, all of the selected light chains had undergone a rearrangement creating highly similar CDRL3 of the same length, hence indicating that combinations very similar to the original pairing were formed in these clones.

Although phage display has been reported to bias the selection of promiscuous heavy chains $^{33}$ and certain allergen-specific IgE $V_{H}$ have been characterized as promiscuous, ${ }^{16,18,34}$ only one of the identified clonotypes in the current study was isolated with a number of unrelated light chains (Table II, clonotype 35). This clonotype was found to dominate the Phl p 5 response and it was also most commonly found among clones selected on Phl p 6. Interestingly though, whereas the Phl p 5-specific paratope could be established from this particular $\mathrm{V}_{\mathrm{H}}$ sequence using a range of light chains with an origin in both $\lambda$ and $\kappa$, only one particular $V_{L}$ created a Phl p 6-reactive binding site (Table II). Despite very similar (Figure 3), and sometimes even identical $\mathrm{V}_{\mathrm{H}}$, these clones had quite different binding behaviors. The Phl p 5-selected clones did not cross-react with Phl p 6, whereas, as noted above, the opposite was true; all the isolated $\mathrm{Phl} \mathrm{p} \mathrm{6-specific} \mathrm{clones} \mathrm{also} \mathrm{recognized} \mathrm{Phl} \mathrm{p} \mathrm{5.} \mathrm{These} \mathrm{findings}$ demonstrate that the ability of this particular $\mathrm{V}_{\mathrm{H}}$ to form a specific binding site for the smaller allergen was much more limited and depended much more on the light chain than was its 


\section{Accepted for publication in the Journal of Allergy and Clinical Immunology (2007)}

DOI:10.1016/j.jaci.2007.06.041

ability to create a Phl p 5-specific binder. However, our investigations neither define which type of light chain that was found in combination with this particular $\mathrm{V}_{\mathrm{H}}$ in the B-cell, nor define precisely which of these two allergens (or their homologues in other grass species) that initially activated its naïve B-cell precursor.

In conclusion, this investigation together with our previous study ${ }^{3}$ has allowed us to connect the overall makeup of the IgE-encoding repertoire with the parts of the repertoire targeting a specific allergen. The results demonstrate that not only is the number of IgEproducing peripheral B-cells during active disease limited, the number of antigens recognized by the IgE repertoire is also highly restricted. These studies have allowed us to pinpoint, at a molecular level, the nature of antibodies with a role in allergy. This type of molecular characterization is a prerequisite for understanding the interplay between the allergen and the antibody response. The strategy further gives the opportunity to understand antibody repertoire development and restrictions as they occur in an individual. We foresee that this approach to characterize specific antibody repertoires will have an impact on allergen vaccine construction and standardization of allergen-specific IgE assays. Furthermore, the isolated antibodies may themselves, following transfer into an appropriate format (e.g. IgG1 or IgG4) be used for passive immunization. 
Accepted for publication in the Journal of Allergy and Clinical Immunology (2007) DOI:10.1016/j.jaci.2007.06.041

\section{ACKNOWLEDGMENTS}

We thank Dr. Johan Lantto for helpful discussion and for critically reading the manuscript. 


\section{REFERENCES}

1. Andersson K, Lidholm J. Characteristics and immunobiology of grass pollen allergens. Int Arch Allergy Immunol 2003; 130:87-107.

2. Mari A. Skin test with a timothy grass (Phleum pratense) pollen extract vs. IgE to a timothy extract vs. IgE to rPhl p 1, rPhl p 2, nPhl p 4, rPhl p 5, rPhl p 6, rPhl p 7, rPhl p 11, and rPhl p 12: epidemiological and diagnostic data. Clin Exp Allergy 2003; 33:43-51.

3. Andreasson U, Flicker S, Lindstedt M, Valenta R, Greiff L, Korsgren M, et al. The human IgE-encoding transcriptome to assess antibody repertoires and repertoire evolution. J Mol Biol 2006; 362:212-27.

4. Davies JM, O'Hehir RE. VH gene usage in immunoglobulin E responses of seasonal rhinitis patients allergic to grass pollen is oligoclonal and antigen driven. Clin Exp Allergy 2004; 34:429-36.

5. Eibensteiner P, Spitzauer S, Steinberger P, Kraft D, Valenta R. Immunoglobulin E antibodies of atopic individuals exhibit a broad usage of VH-gene families. Immunology 2000; 101:112-9.

6. Tilgner J, Golembowski S, Kersten B, Sterry W, Jahn S. VH genes expressed in peripheral blood IgE-producing B cells from patients with atopic dermatitis. Clin Exp Immunol 1997; 107:528-35.

7. Edwards MR, Brouwer W, Choi CH, Ruhno J, Ward RL, Collins AM. Analysis of IgE antibodies from a patient with atopic dermatitis: biased V gene usage and evidence for polyreactive IgE heavy chain complementarity-determining region 3. J Immunol 2002; 168:6305-13. 
8. Janezic A, Chapman CJ, Snow RE, Hourihane JO, Warner JO, Stevenson FK. Immunogenetic analysis of the heavy chain variable regions of IgE from patients allergic to peanuts. J Allergy Clin Immunol 1998; 101:391-6.

9. Snow RE, Chapman CJ, Frew AJ, Holgate ST, Stevenson FK. Analysis of Ig VH region genes encoding IgE antibodies in splenic B lymphocytes of a patient with asthma. J Immunol 1995; 154:5576-81.

10. Snow RE, Djukanovic R, Stevenson FK. Analysis of immunoglobulin E VH transcripts in a bronchial biopsy of an asthmatic patient confirms bias towards VH5, and indicates local clonal expansion, somatic mutation and isotype switch events. Immunology 1999; 98:646-51.

11. de Wildt RM, Hoet RM, van Venrooij WJ, Tomlinson IM, Winter G. Analysis of heavy and light chain pairings indicates that receptor editing shapes the human antibody repertoire. J Mol Biol. 1999; 285:895-901.

12. Efremov DG, Batista FD, Burrone OR. Molecular analysis of IgE H-chain transcripts expressed in vivo by peripheral blood lymphocytes from normal and atopic individuals. J Immunol 1993; 151:2195-207.

13. van der Stoep N, van der Linden J, Logtenberg T. Molecular evolution of the human immunoglobulin E response: high incidence of shared mutations and clonal relatedness among epsilon VH5 transcripts from three unrelated patients with atopic dermatitis. J Exp Med 1993; 177:99-107.

14. Flicker S, Steinberger P, Ball T, Krauth MT, Verdino P, Valent P, et al. Spatial clustering of the IgE epitopes on the major timothy grass pollen allergen $\mathrm{Phl} \mathrm{p} 1$ : importance for allergenic activity. J Allergy Clin Immunol 2006; 117:1336-43. 
15. Flicker S, Steinberger P, Norderhaug L, Sperr WR, Majlesi Y, Valent P, et al. Conversion of grass pollen allergen-specific human IgE into a protective IgG(1) antibody. Eur J Immunol 2002; 32:2156-62.

16. Jakobsen CG, Bodtger U, Kristensen P, Poulsen LK, Roggen EL. Isolation of highaffinity human IgE and IgG antibodies recognising Bet v 1 and Humicola lanuginosa lipase from combinatorial phage libraries. Mol Immunol 2004; 41:941-53.

17. Laukkanen ML, Makinen-Kiljunen S, Isoherranen K, Haahtela T, Söderlund H, Takkinen K. Hevein-specific recombinant IgE antibodies from human single-chain antibody phage display libraries. J Immunol Methods 2003; 278:271-81.

18. Steinberger P, Kraft D, Valenta R. Construction of a combinatorial IgE library from an allergic patient. Isolation and characterization of human IgE Fabs with specificity for the major timothy grass pollen allergen, Phl p 5. J Biol Chem 1996; 271:10967-72.

19. Lindstedt M, Schiött A, Bengtsson A, Larsson K, Korsgren M, Greiff L, et al. Genomic and functional delineation of dendritic cells and memory T cells derived from grass pollen-allergic patients and healthy individuals. Int Immunol 2005; 17:4019.

20. Engberg J, Andersen PS, Nielsen LK, Dziegiel M, Johansen LK, Albrechtsen B. Phage-display libraries of murine and human antibody Fab fragments. Methods Mol Biol 1995; 51:355-76.

21. Giudicelli V, Chaume D, Lefranc MP. IMGT/V-QUEST, an integrated software program for immunoglobulin and T cell receptor V-J and V-D-J rearrangement analysis. Nucleic Acids Res 2004; 32:W435-40.

22. Monod MY, Giudicelli V, Chaume D, Lefranc MP. IMGT/JunctionAnalysis: the first tool for the analysis of the immunoglobulin and T cell receptor complex V-J and V-DJ JUNCTIONs. Bioinformatics 2004; 20 Suppl 1:I379-I85. 


\section{Accepted for publication in the Journal of Allergy and Clinical Immunology (2007)}

\section{DOI:10.1016/j.jaci.2007.06.041}

23. Lefranc MP, Pommie C, Ruiz M, Giudicelli V, Foulquier E, Truong L, et al. IMGT unique numbering for immunoglobulin and T cell receptor variable domains and Ig superfamily V-like domains. Dev Comp Immunol 2003; 27:55-77.

24. Matthiesen F, Friberg L, Olsen M, Lowenstein H. Purification and characterization of the Phleum pratense (timothy) pollen allergen Phl p VI. In: Kraft D, Sehon A, editors. Molecular Biology and Immunology of Allergens. Boca Raton: CRC; 1993. p. 189-91.

25. Petersen A, Bufe A, Schramm G, Schlaak M, Becker WM. Characterization of the allergen group VI in timothy grass pollen (Phl p 6). II. cDNA cloning of Phl p 6 and structural comparison to grass group V. Int Arch Allergy Immunol 1995; 108:55-9.

26. Vrtala S, Fischer S, Grote M, Vangelista L, Pastore A, Sperr WR, et al. Molecular, immunological, and structural characterization of Phl p 6, a major allergen and Pparticle-associated protein from Timothy grass (Phleum pratense) pollen. J Immunol 1999; 163:5489-96.

27. Marknell DeWitt A, Niederberger V, Lehtonen P, Spitzauer S, Sperr WR, Valent P, et al. Molecular and immunological characterization of a novel timothy grass (Phleum pratense) pollen allergen, Phl p 11. Clin Exp Allergy 2002; 32:1329-40.

28. Xu JL, Davis MM. Diversity in the CDR3 region of $\mathrm{V}(\mathrm{H})$ is sufficient for most antibody specificities. Immunity 2000; 13:37-45.

29. Gleich GJ, Jacob GL. Immunoglobulin E antibodies to pollen allergens account for high percentages of total immunoglobulin E protein. Science 1975; 190:1106-8.

30. Niederberger V, Laffer S, Froschl R, Kraft D, Rumpold H, Kapiotis S, et al. IgE antibodies to recombinant pollen allergens (Phl p 1, Phl p 2, Phl p 5, and Bet v 2) account for a high percentage of grass pollen-specific IgE. J Allergy Clin Immunol 1998; 101:258-64. 


\section{DOI:10.1016/j.jaci.2007.06.041}

31. Niederberger V, Ring J, Rakoski J, Jager S, Spitzauer S, Valent P, et al. Antigens drive memory IgE responses in human allergy via the nasal mucosa. Int Arch Allergy Immunol. 2007;142:133-44.

32. Erazo A, Kutchukhidze N, Leung M, Christ AP, Urban JF Jr, Curotto de Lafaille MA, et al. Unique maturation program of the IgE response in vivo. Immunity. 2007; 26:191-203.

33. Meijer PJ, Andersen PS, Haahr Hansen M, Steinaa L, Jensen A, Lantto J, et al. Isolation of human antibody repertoires with preservation of the natural heavy and light chain pairing. J Mol Biol 2006; 358:764-72.

34. Laffer S, Steinberger P, Kraft D, Valenta R. Promiscuous use of light chains by human IgE antibodies specific for three major grass pollen allergens. Int Arch Allergy Immunol 2001; 124:29-30. 


\section{FIGURE LEGENDS}

Figure 1. Serum IgE reactivity of a grass pollen allergic patient, who was utilized for the construction of the combinatorial IgE library used in this study, to various grass pollen allergens; recombinant and natural (as found in timothy extract, Phl p E).

Figure 2. Binding specificity of representative examples of selected scFv as determined by ELISA on different recombinant timothy allergens and on the natural extracts $\mathrm{Phl} p \mathrm{E}$ (timothy) and Lol E (rye grass). The clones have preferred recognition of the substrate used in the selection, except for the clones selected on Phl p 6 (here exemplified by clone p6-1) which show cross-reactivity to Phl p 5.

Figure 3. Sequences of scFv selected on Phl p 6 (clone p6-1) and Phl p 5 (clones p5-9 and p5-13), having essentially the same $\mathrm{V}_{\mathrm{H}}$ (clonotype 35) but differing in the light chain. The CDR of both chains are high-lighted, identical residues are shown with dots and gaps are indicated by dashes. 
Table I. Genetic origin of IgE $\mathrm{V}_{\mathrm{H}}$ from donor $\mathrm{A} 3$, as found among randomly picked transcripts. Previously, based on sequence homology, the 63 randomly sequenced IgE $V_{H}$ transcripts of donor A3 were grouped into 33 different clonotypes (1-33). As indicated in the specificity column, 7 of these clonotypes were identified among the grass pollen specific scFv. New clonotypes, not found among the random transcripts, were also identified among the grass pollen specific antibody fragments (clonotype 34-40). ${ }^{a}$

\begin{tabular}{|c|c|c|c|}
\hline Clonotype & $\begin{array}{l}\text { Number of } \\
\text { identified } \\
\text { members }\end{array}$ & $\begin{array}{l}\text { Heavy chain } \\
\text { gene origin }\end{array}$ & Specificity \\
\hline 1 & 2 & IGHV1-2 & \\
\hline 2 & 1 & IGHV1-2 & \\
\hline 3 & 1 & IGHV1-2 & \\
\hline 4 & 4 & IGHV1-18 & \\
\hline 5 & 1 & IGHV1-18 & Phl p 1 \\
\hline 6 & 1 & IGHV1-58 & \\
\hline 7 & 2 & IGHV1-69 & \\
\hline 8 & 1 & IGHV1-69 & \\
\hline 9 & 2 & IGHV3-7 & \\
\hline 10 & 1 & IGHV3-11 & \\
\hline 11 & 1 & IGHV3-21 & \\
\hline 12 & 2 & IGHV3-21 & \\
\hline 13 & 2 & IGHV3-23 & \\
\hline 14 & 6 & IGHV3-23 & \\
\hline 15 & 1 & IGHV3-23 & \\
\hline 16 & 1 & IGHV3-23 & Phl p 5 \\
\hline 17 & 1 & IGHV3-23 & \\
\hline 18 & 2 & IGHV3-30 & Phl p 11 \\
\hline 19 & 3 & IGHV3-30 & \\
\hline 20 & 1 & IGHV3-30 & \\
\hline 21 & 1 & IGHV3-30 & \\
\hline 22 & 1 & IGHV3-30 & \\
\hline 23 & 1 & IGHV3-43 & \\
\hline 24 & 2 & IGHV3-43 & Phl p 4 \\
\hline 25 & 1 & IGHV3-53 & Phl p 1 \\
\hline 26 & 6 & IGHV4-31 & Phl p 2 \\
\hline 27 & 2 & IGHV4-31 & \\
\hline 28 & 2 & IGHV4-39 & \\
\hline 29 & 4 & IGHV5-a & Phl p 5 \\
\hline 30 & 1 & IGHV5-a & \\
\hline 31 & 1 & IGHV5-51 & \\
\hline 32 & 4 & IGHV7-4 & \\
\hline 33 & 1 & IGHV7-4-1 & \\
\hline 34 & - & IGHV1-18 & Phl p 5 \\
\hline 35 & - & IGHV3-11 & $\mathrm{Phl} \mathrm{p} \mathrm{5/6^{b }}$ \\
\hline 36 & - & IGHV3-11 & $\mathrm{Phl} \mathrm{p} \mathrm{5/6^{ \textrm {c } }}$ \\
\hline 37 & - & IGHV3-21 & $\mathrm{Phl}$ p 5/6 ${ }^{\mathrm{c}}$ \\
\hline 38 & - & IGHV3-23 & Phl p 5 \\
\hline 39 & - & IGHV3-30 & Phl p 11 \\
\hline 40 & - & IGHV4-39 & Phl p 4 \\
\hline
\end{tabular}

${ }^{a}$ An extended version of this table (Table E1) including IGHJ and IGHD gene origin, CDRH3 lengths and GenBank accession numbers is available in the Online Repository at www.jacionline.org.

${ }^{\mathrm{b}}$ All members of this clonotype recognize Phl p 5 and some also recognize Phl p 6.

${ }^{\mathrm{c}}$ Members of this clonotype recognize both Phl p 5 and Phl p 6. 
Accepted for publication in the Journal of Allergy and Clinical Immunology (2007) DOI:10.1016/j.jaci.2007.06.041

Table II. Genetic origin of genes encoding the isolated grass pollen specific scFv. ${ }^{\text {a }}$

\begin{tabular}{|c|c|c|c|}
\hline Clonotype & Specificity & $\begin{array}{c}\text { Heavy chain } \\
\text { gene origin }\end{array}$ & $\begin{array}{l}\text { Light chain } \\
\text { gene origin }\end{array}$ \\
\hline 5 & Phl p 1 & IGHV1-18 & IGLV3-25 \\
\hline 25 & Phl p 1 & IGHV3-53 & IGKV2-28 \\
\hline 26 & Phl p 2 & IGHV4-31 & $\begin{array}{l}\text { IGKV1-5 } \\
\text { IGKV1-12 }\end{array}$ \\
\hline 24 & Phl p 4 & IGHV3-43 & $\begin{array}{l}\text { IGLV1-44 } \\
\text { IGLV1-47 }\end{array}$ \\
\hline 40 & Phl p 4 & IGHV4-39 & IGKV1-6 \\
\hline 16 & Phl p 5 & IGHV3-23 & IGLV3-1 \\
\hline 29 & Phl p 5 & IGHV5-a & IGLV1-44 \\
\hline 34 & Phl p 5 & IGHV1-18 & IGLV1-44 \\
\hline 38 & Phl p 5 & IGHV3-23 & IGLV3-25 \\
\hline 35 & $\begin{array}{c}\text { Phl p } 5 \\
\text { Phl p } 5 \\
\text { Phl p } 5 \\
\text { Phl p } 5 \\
\text { Phl p } 5 \\
\text { Phl p 5/6 } \\
\end{array}$ & IGHV3-11 & $\begin{array}{c}\text { IGKV1-39 } \\
\text { IGLV1-44 } \\
\text { IGLV2-8 } \\
\text { IGLV2-11 } \\
\text { IGLV2-14 } \\
\text { IGLV2-14 } \\
\end{array}$ \\
\hline 36 & Phl p 5/6 & IGHV3-11 & IGLV3-1 \\
\hline 37 & Phl p 5/6 & IGHV3-21 & IGLV2-14 \\
\hline 18 & Phl p 11 & IGHV3-30 & IGLV2-14 \\
\hline 39 & Phl p 11 & IGHV3-30 & IGKV4-1 \\
\hline
\end{tabular}

${ }^{a}$ An extended version of this table (Table E2) including GenBank accession numbers is available in the Online Repository at www.jacionline.org.

${ }^{\mathrm{b}}$ Clone recognizes both Phl p 5 and Phl p 6. 
Accepted for publication in the Journal of Allergy and Clinical Immunology (2007) DOI:10.1016/j.jaci.2007.06.041

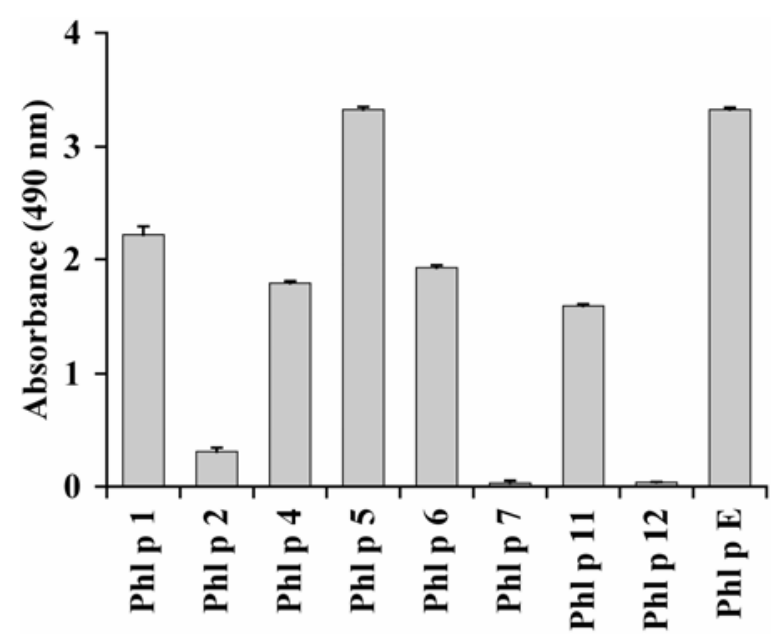

Figure 1 
Accepted for publication in the Journal of Allergy and Clinical Immunology (2007) DOI:10.1016/j.jaci.2007.06.041

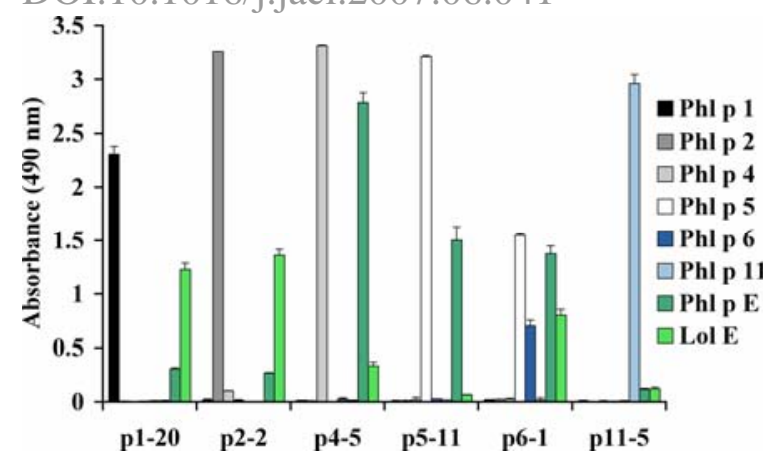

Figure 2 
Accepted for publication in the Journal of Allergy and Clinical Immunology (2007) DOI:10.1016/j.jaci.2007.06.041

\section{Heavy chain}

p6-1 EVQLVQS G G L VK PGG S LRLSCAAS GF IFSDHYMAWVRQT PGKGLEWISY TSMTSSYTNYA

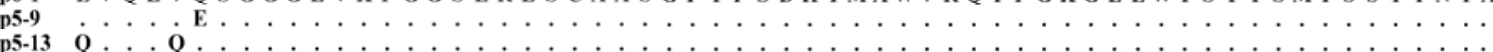

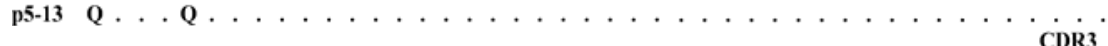

p6-1 DS VKGR F I S R D AKN S LS LEMNS LRVEDTAVYYCARDRLRNWNDDAFDIWGPGTMVTVS

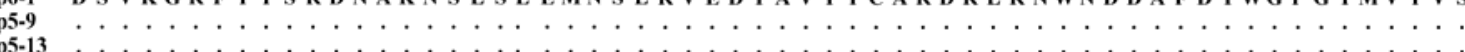

\section{Light chain}

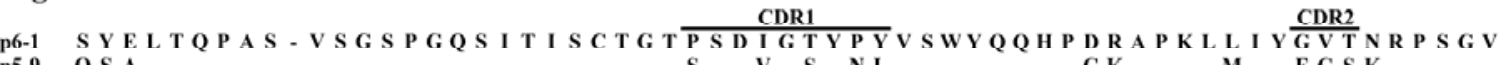

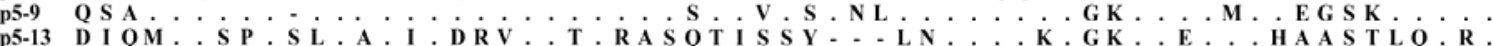

p6-1 S NR F S G S K S G N T A S L T I S ELQA E DEA DYY C S Y T L TS IVVFGGGTKLTVLG

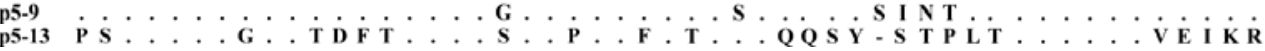

Figure 3 


\section{Online Repository}

\section{Delineating the specificity of an IgE-encoding transcriptome}

Helena Persson, MSc, ${ }^{\text {a }}$ Mardjaneh Karbalaei Sadegh, MSc, ${ }^{a}$ Lennart Greiff, MD, ${ }^{\text {b }}$ and Mats Ohlin, $\mathrm{PhD}^{\mathrm{a} *}$

${ }^{a}$ Department of Immunotechnology, Lund University, BMC D13, SE-221 84 Lund, Sweden

${ }^{\mathrm{b}}$ Department of Otorhinolaryngology, Lund University Hospital, SE-221 85 Lund, Sweden

*To whom correspondence should be addressed:

E-mail address: mats.ohlin@immun.lth.se

Tel: +46-46-222 4322

Fax: +46-46-222 4200 


\section{Accepted for publication in the Journal of Allergy and Clinical Immunology (2007)}

DOI:10.1016/j.jaci.2007.06.041

Table E1. Genetic origin of IgE $\mathrm{V}_{\mathrm{H}}$ from donor A3, as found among randomly picked transcripts. Previously, based on sequence homology, the 63 randomly sequenced $\operatorname{IgE} \mathrm{V}_{\mathrm{H}}$ transcripts of donor A3 were grouped into 33 different clonotypes (1-33). As indicated in the specificity column, 7 of these clonotypes were identified among the grass pollen specific scFv. New clonotypes, not found among the random transcripts, were also identified among the grass pollen specific antibody fragments (clonotype 34-40).

\begin{tabular}{|c|c|c|c|c|c|c|c|}
\hline \multirow[b]{2}{*}{ Clonotype } & \multirow[b]{2}{*}{$\begin{array}{l}\text { Number of } \\
\text { identified } \\
\text { members }\end{array}$} & \multicolumn{3}{|c|}{ Heavy chain gene origin } & \multirow[b]{2}{*}{$\begin{array}{c}\text { CDRH3 } \\
\text { length }\end{array}$} & \multirow[b]{2}{*}{$\begin{array}{l}\text { GenBank accession nos. } \\
\text { of random } V_{H} \text { transcripts } \\
(\mathrm{DQ065XXX)})\end{array}$} & \multirow[b]{2}{*}{ Specificity } \\
\hline & & IGHV & IGHJ & IGHD & & & \\
\hline 1 & 2 & $1-2$ & 4 & $1-26$ & 13 & 683,691 & \\
\hline 2 & 1 & $1-2$ & 4 & $4-23$ & 13 & 697 & \\
\hline 3 & 1 & $1-2$ & 6 & $3-10$ & 20 & 690 & \\
\hline 4 & 4 & $1-18$ & 4 & $3-22$ & 14 & $675,679,688,695$ & \\
\hline 5 & 1 & $1-18$ & 4 & $1-26$ & 7 & 722 & Phl p 1 \\
\hline 6 & 1 & $1-58$ & 6 & $3-3$ & 19 & 681 & \\
\hline 7 & 2 & $1-69$ & 4 & $2-8$ & 12 & 677,701 & \\
\hline 8 & 1 & $1-69$ & 4 & 6-19 & 12 & 705 & \\
\hline 9 & 2 & $3-7$ & 6 & $3-3$ & 18 & 716,724 & \\
\hline 10 & 1 & $3-11$ & 6 & $3-9$ & 24 & 715 & \\
\hline 11 & 1 & $3-21$ & 4 & $2-2$ & 9 & 704 & \\
\hline 12 & 2 & $3-21$ & 4 & $3-3$ & 14 & 668,694 & \\
\hline 13 & 2 & $3-23$ & 4 & $3-22$ & 18 & 713,723 & \\
\hline 14 & 6 & $3-23$ & 6 & 3-16 & 15 & $672,678,685,693,721,728$ & \\
\hline 15 & 1 & $3-23$ & 4 & $1-26$ & 12 & 684 & \\
\hline 16 & 1 & $3-23$ & 4 & $2-15$ & 16 & 707 & Phl p 5 \\
\hline 17 & 1 & $3-23$ & 1 & 6-19 & 15 & 708 & \\
\hline 18 & 2 & $3-30$ & 4 & $3-22$ & 18 & 718,725 & Phl p 11 \\
\hline 19 & 3 & $3-30$ & 4 & $3-22$ & 16 & $674,689,709$ & \\
\hline 20 & 1 & $3-30$ & 6 & $2-15$ & 26 & 669 & \\
\hline 21 & 1 & $3-30$ & 2 & $3-9$ & 17 & 696 & \\
\hline 22 & 1 & $3-30$ & 4 & $3-22$ & 17 & 692 & \\
\hline 23 & 1 & $3-43$ & 4 & $3-9$ & 20 & 726 & \\
\hline 24 & 2 & $3-43$ & 3 & 6-19 & 12 & 670,719 & Phl p 4 \\
\hline 25 & 1 & $3-53$ & 4 & 3-16 & 11 & 720 & Phl p 1 \\
\hline 26 & 6 & $4-31$ & 4 & 3-10 & 10 & $666,680,686,711,712,714$ & Phl p 2 \\
\hline 27 & 2 & $4-31$ & 4 & 6-19 & 14 & 700,706 & \\
\hline 28 & 2 & $4-39$ & 4 & $3-10$ & 10 & 676,717 & \\
\hline 29 & 4 & 5-a & 4 & $2-15$ & 20 & $702,703,710,727$ & Phl p 5 \\
\hline 30 & 1 & 5-a & 3 & 6-19 & 14 & 698 & \\
\hline 31 & 1 & $5-51$ & 4 & $6-13$ & 12 & 673 & \\
\hline 32 & 4 & $7-4$ & 4 & $3-22$ & 15 & 667, 671, 682, 699, & \\
\hline 33 & 1 & $7-4-1$ & 4 & $3-3$ & 17 & 687 & \\
\hline 34 & - & $1-18$ & 6 & 3-22 & 23 & - & Phl p 5 \\
\hline 35 & - & 3-11 & 3 & $1-1$ & 15 & - & Phl p 5/6 \\
\hline 36 & - & 3-11 & 4 & $3-22$ & 9 & - & Phl p 5/6 \\
\hline 37 & - & $3-21$ & 4 & $6-13$ & 13 & - & Phl p 5/6 \\
\hline 38 & - & $3-23$ & 6 & $3-3$ & 21 & - & Phl p 5 \\
\hline 39 & - & $3-30$ & 4 & 3-22 & 16 & - & Phl p 11 \\
\hline 40 & - & $4-39$ & 4 & $4-4$ & 24 & - & Phl p 4 \\
\hline
\end{tabular}

${ }^{\mathrm{a}}$ All members of this clonotype recognize Phl p 5 and some also recognize $\mathrm{Phl} \mathrm{p} 6$.

${ }^{\mathrm{b}}$ Members of this clonotype recognize both Phl p 5 and Phl p 6. 
DOI:10.1016/j.jaci.2007.06.041

Table E2. Genetic origin of genes encoding the isolated grass pollen specific scFv.

\begin{tabular}{|c|c|c|c|c|}
\hline Clonotype & Specificity & $\begin{array}{l}\text { Heavy chain } \\
\text { gene origin }\end{array}$ & $\begin{array}{l}\text { Light chain } \\
\text { gene origin }\end{array}$ & GenBank accession nos. \\
\hline 5 & Phl p 1 & IGHV1-18 & IGLV3-25 & EF607095-6 \\
\hline 25 & Phl p 1 & IGHV3-53 & IGKV2-28 & EF607094 \\
\hline 26 & Phl p 2 & IGHV4-31 & $\begin{array}{l}\text { IGKV1-5 } \\
\text { IGKV1-12 }\end{array}$ & $\begin{array}{c}\text { EF607100 } \\
\text { EF607097-9, EF607101-2 }\end{array}$ \\
\hline 24 & Phl p 4 & IGHV3-43 & $\begin{array}{l}\text { IGLV1-44 } \\
\text { IGLV1-47 }\end{array}$ & $\begin{array}{l}\text { EF607108-9 } \\
\text { EF607103-7 }\end{array}$ \\
\hline 40 & Phl p 4 & IGHV4-39 & IGKV1-6 & EF607110 \\
\hline 16 & Phl p 5 & IGHV3-23 & IGLV3-1 & EF601887 $^{\mathrm{b}}$ \\
\hline 29 & Phl p 5 & IGHV5-a & IGLV1-44 & DQ065730 \\
\hline 34 & Phl p 5 & IGHV1-18 & IGLV1-44 & EF607117 \\
\hline 38 & Phl p 5 & IGHV3-23 & IGLV3-25 & EF607113 \\
\hline 35 & $\begin{array}{l}\text { Phl p } 5 \\
\text { Phl p } 5 \\
\text { Phl p } 5 \\
\text { Phl p } 5 \\
\text { Phl p } 5 \\
\text { Phl p 5/6 }\end{array}$ & IGHV3-11 & $\begin{array}{l}\text { IGKV1-39 } \\
\text { IGLV1-44 } \\
\text { IGLV2-8 } \\
\text { IGLV2-11 } \\
\text { IGLV2-14 } \\
\text { IGLV2-14 }\end{array}$ & $\begin{array}{c}\text { EF607116, DQ065729 } \\
\text { EF607118 } \\
\text { EF607115 } \\
\text { EF607119 } \\
\text { EF607112, EF607114, DQ065733 } \\
\text { EF607120 }\end{array}$ \\
\hline 36 & Phl p 5/6 ${ }^{\mathrm{a}}$ & IGHV3-11 & IGLV3-1 & EF607122 \\
\hline 37 & Phl p 5/6 & IGHV3-21 & IGLV2-14 & EF607121, EF607123-4 \\
\hline 18 & Phl p 11 & IGHV3-30 & IGLV2-14 & EF607125-6, EF607128 \\
\hline 39 & Phl p 11 & IGHV3-30 & IGKV4-1 & EF607127 \\
\hline
\end{tabular}

${ }^{\mathrm{a}}$ Clone recognizes both $\mathrm{Phl} \mathrm{p} 5$ and $\mathrm{Phl}$ p 6.

${ }^{\mathrm{b}}$ Clone isolated from the same library but in a separate study (J. Persson et al., manuscript in preparation).

${ }^{\mathrm{c}}$ Clone isolated from the same library but in a separate study. ${ }^{\mathrm{E} 1}$ 


\section{REFERENCES}

E1. Andreasson U, Flicker S, Lindstedt M, Valenta R, Greiff L, Korsgren M, et al. The human IgE-encoding transcriptome to assess antibody repertoires and repertoire evolution. J Mol Biol 2006; 362:212-27. 